\title{
Synthesis and characterization, by high pressure neutron and X-ray powder diffraction, of hybrid perovskites containing helium as a structural component
}

\author{
Angus P. Wilkinson ${ }^{a}$ and Brett R. Hester ${ }^{b}$ \\ a School of Chemistry and Biochemistry, Georgia Institute of Technology, Atlanta, GA 30332, \\ USA, angus.wilkinson@chemistry.gatech.edu \\ b School of Chemistry and Biochemistry, Georgia Institute of Technology, Atlanta, GA 30332, \\ USA, bhester7@gatech.edu
}

\begin{abstract}
$\mathrm{ReO}_{3}$-type mixed metal fluorides, $\mathrm{ABF}_{6}$, have been explored for their potential as low and negative thermal expansion materials (NTE). For example, CaZrF ${ }_{6}$ displays strong NTE, $\alpha_{V}=$ $-53 \mathrm{ppmK}^{-1}$ at $100 \mathrm{~K}$, and remain cubic over a wide temperature range [1]. Most cubic $\mathrm{ReO}_{3}-$ type materials, including $\mathrm{CaZrF}_{6}$, undergo symmetry lowering phase transitions below $1 \mathrm{GPa}$, which can be an obstacle to their application. In order to further our understanding of how $\mathrm{CaZrF}_{6}$ behaves as a function of temperature and pressure, a variable temperature and pressure neutron diffraction study was conducted using helium as the pressure medium. Surprisingly, the unit cell volume was seen to increase on compression at room temperature. This is attributed to the insertion of helium into $\mathrm{CaZrF}_{6}$. The behaviour of the lattice constants as a function of temperature and pressure, combined with Rietveld refinement of helium site occupancies, indicate that He is inserted into the empty "A-sites" of the pseudo-perovskite starting structure. The helium is trapped in the structure when cooled below $190 \mathrm{~K}$ at pressure. Gas uptake and release experiments indicate that pressurizing $\mathrm{CaZrF}_{6}$ to $\sim 500 \mathrm{MPa}$ results in $\sim 55 \%$ filling of the empty A sites leading to a defect perovskite $\mathrm{He}_{2-x}[\mathrm{CaZr}] \mathrm{F}_{6}$. Compression in nitrogen leads to different behaviour. The volume of the material decreases steadily leading to a bulk modulus estimate of $36.7 \mathrm{GPa}$, which is consistent with earlier measurements using a DAC and a silicone oil medium. The inclusion of helium on the A-site of $\mathrm{CaZrF}_{6}$ at $\sim 450$ $\mathrm{MPa}$, reduces, but does not completely suppress, the material's NTE. High pressure x-ray diffraction studies show that helium insertion stabilizes the perovskite structure to pressures greater than $3 \mathrm{GPa}$ and suggest that the stoichiometric helium containing perovskite can be formed at less than $1 \mathrm{GPa}$. If all of the A-sites in $\mathrm{CaZrF}_{6}$ could be completely filled with hydrogen, rather than helium, the volumetric storage density would be $\sim 0.044 \mathrm{~kg} \mathrm{H}_{2} / \mathrm{L}$ [2].
\end{abstract}

\section{References}

[1] Hancock J.C., Chapman K.W., Halder G.J., Morelock C.R., Kaplan B.S., Gallington L.C., Bongiorno A., Han C., Zhou S., Wilkinson A.P., Chem. Mater. 27 (2015) 3912-3918.

[2] Hester B.R., dos Santos A.M., Molaison J.J., Hancock, J.C., Wilkinson A.P., J. Am. Chem. Soc. 139 (2017) 13284-13287. 